\title{
An Investigation of Be/X-ray Pulsars with OGLE-III Data
}

\author{
P.C. Schmidtke \& A.P. Cowley \\ Dept. of Physics \& Astronomy, Arizona State Univ., Tempe, AZ, 85287-1504, USA; \\ paul.schmidtke@asu.edu, anne.cowley@asu.edu \\ and \\ A. Udalski \\ Warsaw University Observatory, Al. Ujazdowskie 4, 00-478 Warsaw, Poland; \\ udalski@astrouw.edu.pl
}

Received —_; accepted _ 


\begin{abstract}
We have studied five seasons of OGLE-III data for eight SMC Be/X-ray pulsars for which no other survey data were available. We have determined orbital periods for four of these binary systems, one of which also shows nonradial pulsations. Optical identification of SMC X-2 is reconsidered, but no periods were found for either of the two possible candidates.
\end{abstract}

Subject headings: X-rays: binaries - stars: Be - (stars:) pulsars - stars: variable - stars: individual: RX J0052.9-7158, 2E 0053.7-7227, SMC X-2, RX J0057.8-7202, CXOU J005750.3-720756, RX J0059.2-7138, XTE J0111.2-7317, RX J0117.6-7330 


\section{Introduction}

Be/X-ray binary systems account for about half of the high-mass X-ray binaries, but relatively little is known about their properties. We have been studying the photometric behavior of these systems in the Small Magellanic Cloud using the OGLE-II and MACHO databases (Szymanski 2005, Udalski et al. 1997, Zebrun et al. 2001, Alcock et al. 1999) in an attempt to learn more about the component stars. Many of these systems contain X-ray pulsars; lists of such systems are given by Coe et al. (2005), Liu et al. (2005), Haberl \& Pietsch (2004), Yokogawa et al. 2003, and others. Some of these binaries lie outside the fields of the OGLE-II and MACHO surveys and hence had no available longterm photometry.

The OGLE-III project covers a larger area around the SMC and includes some Be/X-ray binaries for which longterm photometry had not previously been obtained. We have undertaken a study of some of these systems, as listed in Table 1 . We give the OGLE-III positions of the optical counterparts that are more accurate, in most cases, than previously available positions. Table 1 also lists the catalogue number in the list of $\mathrm{H} \alpha$ emission-line stars in the SMC by Meyssonnier \& Azzopardi (1993; denoted as [MA93]) and the SXP number (Coe et al. 2005) which gives the X-ray pulse period for S mall Magellanic Cloud X-ray Pulsars. Finding charts for all systems studied here can be found on M. Coe's web site ${ }^{1}$. However, because of confusion about the optical identification of SMC X-2, we present a new finding chart in this paper.

\footnotetext{
${ }^{1}$ http://www.astro.soton.ac.uk/ mjc/smc
} 


\section{Analysis of Data from OGLE-III}

The OGLE-III data cover five years with photometry in the $I$ bandpass. Fig. 1 displays the longterm $I$ light curves for the sources studied here. For all systems the data were prewhitened (flattened) using a low-order polynomial fit to remove long term variations in brightness. In some cases it was necessary to flatten the data in segments when there were large, irregular brightness changes (e.g. SMC X-2). We use $I^{*}$ to designate the flattened data. To analyze the $I^{*}$ data we computed periodograms using the technique described by Horne \& Baliunas (1986). When the variations are very non-sinusoidal we also used the phase dispersion minimization (PDM) technique of Stellingwerf (1978). We searched for photometric periods in the range of 0.25 days to 1000 days. The techniques we used here are more fully described in our earlier papers on Be/X-ray pulsar binaries (e.g. Schmidtke et al. 2004, Schmidtke \& Cowley 2005, Schmidtke \& Cowley 2006).

\section{Individual Be/X-ray Pulsar Systems Studied with OGLE-III Data}

\section{1. $\quad$ RX J0052.9-7158 = SXP169}

This pulsar is identified with the Be star [MA93]623. Galache et al. (2005) note that SXP169 and SXP165 are the same pulsar which has been spun up by $\sim 3$ sec over 7.5 years.

The OGLE-III light curve for SXP169 (Fig. 1) shows a small longterm variation with considerable scatter about the mean. Analysis of all seasons of photometry shows two clear periods. The first is the orbital period of $\mathrm{P}=67.6 \pm 0.3$ days, found both in the periodogram and in the PDM variance (Fig. 2). The epoch of maximum is JD 2452583.3. The light curve folded on this ephemeris shows an outburst of $\sim 0.02$ mag with a rapid rise and more gradual decline. Galache et al. (2005) derived a period of $68.6 \pm 0.2$ days from their weekly

X-ray monitoring program with $R X T E$. We note that both our period and outburst epoch 
are in reasonable agreement with those of Galache et al.

In addition to orbital outbursts, we also have found nonradial pulsations (NRP) of the Be star with a period of $\mathrm{P}_{N R P}=0.755$ days. These pulsations are best detected in the out-of-outburst data (i.e. from orbital phases $\sim 0.2-0.9$. In Fig. 2 we plot the periodogram and folded light curve for these NRP. There is no evidence that the pulsation period changed through the five seasons of OGLE-III observations.

\section{2. $\quad \mathrm{SMC} \mathrm{X-2} \mathrm{=} \mathrm{RX} \mathrm{J0054.5-7340} \mathrm{=} \mathrm{SXP2.37}$}

SMC X-2 is one of the earliest X-ray sources found in the SMC (Li, Jernigan, \& Clark 1977), and it was soon noticed to be highly variable (Li \& Clark 1977). Sanduleak \& Philip (1977) identified SMC X-2 with a 14th magnitude OB star, and this was supported by spectroscopic observations by Allen (1977) who found H emission lines. Subsequent spectra taken by Crampton et al. (1978) showed the star to be a late Oe star with a velocity appropriate to the SMC. However, Murdin, Morton, \& Thomas (1979) later obtained an improved image of the field and resolved the optical counterpart into a close double with $\sim 0.8$ mag difference in brightness. They assigned spectral types of $\mathrm{O}$ to the brighter (northern) star and Be to the fainter (southern) star. Because the components are very close together $\left(\sim 2.5^{\prime \prime}\right)$, it is possible that the emission observed by both Allen and by Crampton et al. came from the fainter component which would have been blended with the spectrum of the brighter star. However, Oe and Be stars have long been known to lose their emission lines from time to time, so we cannot rule out the brighter component as being the X-ray source. The X-ray position is not sufficiently accurate to distinquish between these two close stars. Because the only finding chart in the literature is a low resolution image (Clark et al. 1978), we present the OGLE-III image in Fig. 3 which clearly shows the two components. In addition, we give the OGLE-III position of each component in Table 1. 
Fig. 1 shows the light curves, using comparable scales, for both the northern (brighter) and southern (fainter) component. The northern star has a swooping light curve with a range of $\sim 1$ mag. This type of variation is commonly seen among the Be/X-ray pulsar binaries. The southern component is 1-2 magnitudes fainter and shows only a small light variation. Because the two components are so close, it is likely that the "variability" of the southern star is due to contamination with the much brighter northern component whose magnitude is extremely variable.

For completeness, we have searched for periodic behavior in both components. The brighter, northern component shows weak periodicities at $\sim 90,46$, and 23 days. All are probably aliases of each other. The folded light curves show very small sinusoidal variation. None is convincing enough to claim as a true orbital period. No periods were found in the analysis of the fainter, southern star. Based on the character of the light curves, we conclude the northern star is more likely to be the optical counterpart of SMC X-2, but an improved X-ray position is needed to confirm this.

\section{3. $\quad 2 \mathrm{E} 0053.7-7227=\mathrm{SXP} 34.1$}

This source was found to be a 34-sec X-ray pulsar by Edge et al. (2004) using Chandra data (CXOU J005527.9-721058). The excellent X-ray position makes the optical identification secure. As can be seen in Fig. 1, the longterm light curve shows a relatively constant mean magnitude of $I=16.82$ with a scatter of $\sim 0.06$ mag. We searched the entire data set and each of the individual seasons for possible photometric periods, but found nothing convincing. 


\section{4. $\quad$ RX J0057.8-7202 $=$ SXP280}

This source is identified with the Be star [MA93]1036. Its longterm light curve (Fig. 1) clearly shows the star undergoes repeated outbursts of $\sim 0.05$ mag. The outburst (orbital) period is $127.3 \pm 1.0$ days with the epoch of maximum light at JD 2452195.2. The periodogram and folded light curve are plotted in Fig. 4. We also searched for shorter periods in the data, using both the entire data set and only the data from phases between outbursts. No clear short periods were identified.

\section{5. $\quad$ CXOU J005750.3-720756 $=$ SXP152}

Macomb et al. (2003) found this source to be a 152-sec X-ray pulsar using Chandra data. The position is coincident with the Be star [MA93]1038. Its OGLE-III longterm light curve (Fig. 1) shows a gradual rise from $I=15.38$ to 15.32 over four years, with considerable scatter in the last two seasons. Analysis of the flattened light curve shows no clear periods. Some sections of the data have power near 70, 130, and 200 days, but these may be artifacts due to the length of data train or spacing between seasons. We were unable to find any consistent period which fit all of the data.

\section{6. $\quad$ RX J0059.2-7138 $=$ SXP2.76}

The longterm light curve for this short-period pulsar is shown in Fig. 1. Even before formal analysis one can detect repeated low-amplitude outbursts. The data were flattened in two segments (first three seasons and final two seasons). Both the individual segments and the full data set give $\mathrm{P}=82.1 \pm 0.4$ days, with the epoch of maximum at JD 2452189.4. The outburst amplitude is $\sim 0.02 \mathrm{mag}$, with some variation from cycle to cycle. The folded light curve (Fig. 5) shows a rapid rise and slower decline. We have also searched for shorter 
periods using both the full data set and data from out-of-outburst phases, but we found no significant short periods that might be due to NRP.

Based on Corbet's (1984) relation between pulse and orbital periods, this source's orbital period is much longer than would be expected. We suggest that the pulsar may have been spun up by accretion of material from the Be star's disk.

\subsection{XTE J0111.2-7317 = SXP31.0}

This X-ray transient was identified by Coe et al. (2000) with a B0-2 emission-line star in the SMC. They give $I=15.31$ on 1999 January 21, which is in general agreement with the range of magnitudes ( $I=15.1-15.3)$ observed in the OGLE-III survey. Coe et al. found an irregular, small emission nebula surrounding the star which they suggest might be a supernova remnant. Covino et al. (2001) obtained spectra and found this star to have hydrogen emission lines, confiming its identification as a Be star.

Analysis of the OGLE-III photometry reveals a pronounced orbital period at $\mathrm{P}=90.4 \pm 0.5$ days, with an epoch of maximum at JD 2452808.9. The periodogram and PDM variance are plotted in Fig. 6, with both clearly showing this orbital period. However, the PDM variance gives the better value since the light curve is very non-sinusoidal. The folded light curve has an extremely rapid climb to peak outburst and a more gradual decay. We have searched the inter-outburst data for any shorter periods, but found none.

\section{8. $\quad$ RX J0117.6-7330 = SXP22.1}

Macomb et al. (1999) found this X-ray transient to be a 22-sec pulsar. It is identified with the Be Star [MA93]1845. Soria (1999) studied the optical counterpart before the 
source was know to contain a pulsar. He gives the spectral type as B0.5IIIe with mean magnitudes of $V=14.9 \pm 0.01$ and $I=14.10 \pm 0.01$. We show the longterm OGLE-III light curve in Fig. 1. It is what we would call a "moderate swooper", with longterm variations from $I \sim 13.9$ to 14.1 over several years.

Analysis of the data from the first three seasons (the part which is most easily flattened) reveals no clear periods that are not related to the length of the data train or to seasonal gaps in the data. The last two seasons were more difficult to prewhiten, but these also did not show any significant periods. Finally, all of the flattened data were combined, but again no photometric periods were found.

\section{Summary}

In summary, we have studied the OGLE-III light curves of eight Be/X-ray pulsars in the SMC. We have found orbital periods for four systems. The folded light curves all show asymmetrical outbursts with the rise time being shorter than the decline. One of the sources (SXP169) shows both orbital outbursts and strong nonradial pulsations. For the remaining stars, no clear periodic behavior was found in the range $0.25-1000$ days. The light curves of two stars near the position of SMC X-2 indicate that the northern (brighter) object is the likely optical counterpart.

In a recent paper on Be/X-ray binaries (Schmidtke \& Cowley 2006) we presented some preliminary results about the photometric behavior of this type of source. The same general trends persist in the present investigation. We find that systems with strong orbital outbursts usually do not exhibit nonradial pulsations of the Be star, and conversely the sources with strong NRP rarely show orbital outbursts. SXP169 is the single exception to this. We also find that systems with "swooping" light curves may show weak orbital 
outbursts, but don't have NRP.

The strength of the orbital outbursts may be related to the eccentricity of the orbit, if the pulsar penetrates the Be star's disk near periastron. Perhaps the NRP sources are in fairly circular orbits with little interaction between the stars, and hence orbital outbursts are not present. To investigate these ideas, spectroscopic studies are needed. Finally, we note that Corbet's (1984) relation between pulse period and orbital period is generally supported here, but it shows considerable scatter. Of note is SXP2.76 which shows an unexpectedly long orbital period $\left(\mathrm{P}_{\text {orb }}=82.1\right.$ days $)$ for its short X-ray pulse period, perhaps indicating that the pulsar has been spun up.

Support for the OGLE project was provided by the following grants: Polish MEiN grant 2P03D02124, NSF grant AST-0204908, and NASA grant NAG5-12212. 


\section{REFERENCES}

Alcock, C., et al. 1999, PASP, 111, 1539

Allen, D. 1977, IAU Circ. \#3143

Clark, G., Doxsey, R., Li, F., Jernigan, J.G., \& van Paradijs, J. 1978, ApJ, 221, L37

Coe, M.J., Haigh, N.J., \& Reig, P. 2000, MNRAS, 314, 290

Coe, M.J., Edge, W.R.T., Galache, J.L., \& McBride, V.A. 2005, MNRAS, 356, 502

Corbet, R.H.D. 1984, A\&A, 141, 91

Covino, S., et al. 2001, A\&A, 374, 1009

Crampton, D., Hutchings, J.B., \& Cowley, A.P. 1978, ApJ, 223, L79

Edge, W.R.T., Coe, M.J., \& McBride, V.A. 2004, ATel, \#217

Galache, J.L., Corbet, R.H.D., Coe, M.J., Laycock, S., Markwardt, C.B., \& Marshall, F.E. 2005, ATel, \#674

Haberl, F., \& Pietsch, W. 2004, A\&A, 414, 667

Horne, J.H., \& Baliunas, S.L. 1986, ApJ, 302, 757

Li, F., \& Clark, G. 1977, IAU Circ. 3154

Li, F., Jernigan, G., \& Clark, G. 1977, IAU Circ. \#3126

Liu, Q.Z., van Paradijs, J., \& van den Heuvel, E.P.J. 2005, A\&A, 442, 1135

Macomb, D.J., Finger, M.H., Harmon, B.A., Lamb, R.C., \& Prince, T.A. 1999, ApJ, 518, L99

Macomb, D.J., Fox, D.W., Lamb, R.C., \& Prince, T.A. 2003, ApJ, 584, L79

Meyssonnier, N., \& Azzopardi, M. 1993, A\&AS, 102, 451 ([MA93])

Murdin, P., Morton, D.C., \& Thomas, R.M. 1979, MNRAS, 186, 42p 
Sanduleak, N., \& Philip, A.G.D. 1977, IAU Circ. \#3127

Schmidtke, P.C., \& Cowley, A.P. 2005, AJ, 130, 2220

Schmidtke, P.C., \& Cowley, A.P. 2006, AJ, submitted

Schmidtke, P.C., Cowley, A.P., Levenson, L., \& Sweet, K. 2004, AJ, 127, 3388

Soria, R. 1999, PASA, 16, 147

Stellingwerf, R.F. 1978, ApJ, 224, 953

Szymanski, M.K. 2005, Acta Astron., 55, 43

Udalski, A., Kubiak, M., \& Szymanski, M. 1997, Acta Astron., 47, 319

Yokogawa, J., Imanishi, K., Tsujimoto, M., Koyama, K., \& Nishiuchi, M. 2003, PASJ, 55, 161

Zebrun, K., et al. 2001, Acta Astron., 51, 317 
Fig. 1.- Longterm OGLE-III light curves of all the Be/X-ray pulsar systems studied in this paper. In the case of SMC X-2, for which the identification is still uncertain, we have included the light curves for both components. The northern star is a "swooper", a photometric behavior which is found in many sources of this type.

Fig. 2.- (top) PDM variance (upper; right scale) and periodogram (lower; left scale) for RX J0052.9-7158 (SXP169). (second) Light curve folded on the orbital period of 67.6 days and binned in 20 phase bins. (third) Periodogram of non-outburst data revealing the strong NRP at 0.755 days. (bottom) Light curve of non-outburst data folded on $\mathrm{P}=0.755$ days.

Fig. 3.- Finding chart for SMC X-2 (SXP2.37) showing two stars which are separated by $\sim 2.5^{\prime \prime}$. North is at the top; east is on the left. The figure is $\sim 1.3^{\prime}$ on each side. The light curves of both components are shown in Fig. 1. See discussion of the optical identification in the text.

Fig. 4.- (top) Periodogram of RX J0057.8-7202 (SXP280) showing the strong period at 127.3 days. The outbursting behavior is clearly visible even without formal analysis (see Fig. 1). (bottom) Light curve folded on the orbital period of $\mathrm{P}=127.3$ days.

Fig. 5.- (top) Periodogram of RX J0059.2-7138 (SXP2.76) showing power at $\mathrm{P}=82.1$ days. (bottom) Light curve folded on the orbital period of $\mathrm{P}=82.1$ days.

Fig. 6.- (top) PDM variance (upper; right scale) and periodogram (lower; left scale) for XTE J0111.2-7317 (SXP31.0). Both techniques show power at $\mathrm{P}=90.4$ days which appears to be the orbital period. (bottom) Light curve folded on the orbital period, showing prominent outbursts. 
Table 1. Small Magellanic Cloud Be/Neutron-Star Systems Studied

\begin{tabular}{lllll}
\hline \hline \multicolumn{1}{c}{ System Name } & SXP \# & {$[\text { MA93 }]^{\mathrm{a}}$} & \multicolumn{2}{c}{2000 Position $^{\mathrm{b}}$} \\
\hline RX J0052.9-7158 & SXP169 & 623 & $00: 52: 55.28$ & $-71: 58: 06.0$ \\
SMC X-2 (North) & SXP2.37 & --- & $00: 54: 33.47$ & $-73: 41: 01.1$ \\
SMC X-2 (South) & SXP2.37 & --- & $00: 54: 33.37$ & $-73: 41: 03.5$ \\
2E 0053.7-7227 & SXP34.1 & --- & $00: 55: 28.46$ & $-72: 10: 56.5$ \\
RX J0057.8-7202 & SXP280 & 1036 & $00: 57: 49.58$ & $-72: 02: 35.7$ \\
CXOU J005750.3-720756 & SXP152 & 1038 & $00: 57: 50.38$ & $-72: 07: 55.9$ \\
RX J0059.2-7138 & SXP2.76 & --- & $00: 59: 12.74$ & $-71: 38: 44.8$ \\
XTE J0111.2-7317 & SXP31.0 & --- & $01: 11: 08.53$ & $-73: 16: 46.0$ \\
RX J0117.6-7330 & SXP22.1 & 1845 & $01: 17: 40.18$ & $-73: 30: 50.5$ \\
\hline
\end{tabular}

${ }^{a}$ From catalogue of SMC H $\alpha$ emission-line stars by Meyssonnier \& Azzopardi (1993).

b2000 positions are from OGLE-III data. 
Table 2. Photometric Periods for SMC Be/Neutron-Star Systems

\begin{tabular}{cccc}
\hline \hline System Names & $\mathrm{P}_{\text {orb }}$ & $\mathrm{NRP}^{\mathrm{a}}$ & References \\
& (days) & (days) & \\
\hline & & & Galache et al. $^{\mathrm{b}}$ \\
RX J0052.9-7158 (SXP169) & 67.6 & 0.755 & \\
RX J0057.8-7202 (SXP280) & 127.3 & - & \\
RX J0059.2-7138 (SXP2.76) & 82.1 & - & Coe et al. ${ }^{\mathrm{c}}$ \\
XTE J0111.2-7317 (SXP31.0) & 90.4 & & \\
\hline
\end{tabular}

${ }^{\mathrm{a}} \mathrm{NRP}=$ nonradial pulsations of the Be star.

${ }^{\mathrm{b}}$ Galache et al. (2005) derived $\mathrm{P}_{\text {orb }}=68.6$ days from recurring X-ray outbursts.

${ }^{\mathrm{c}}$ Coe et al. (2000) found a small emission nebula surrounding this system. 

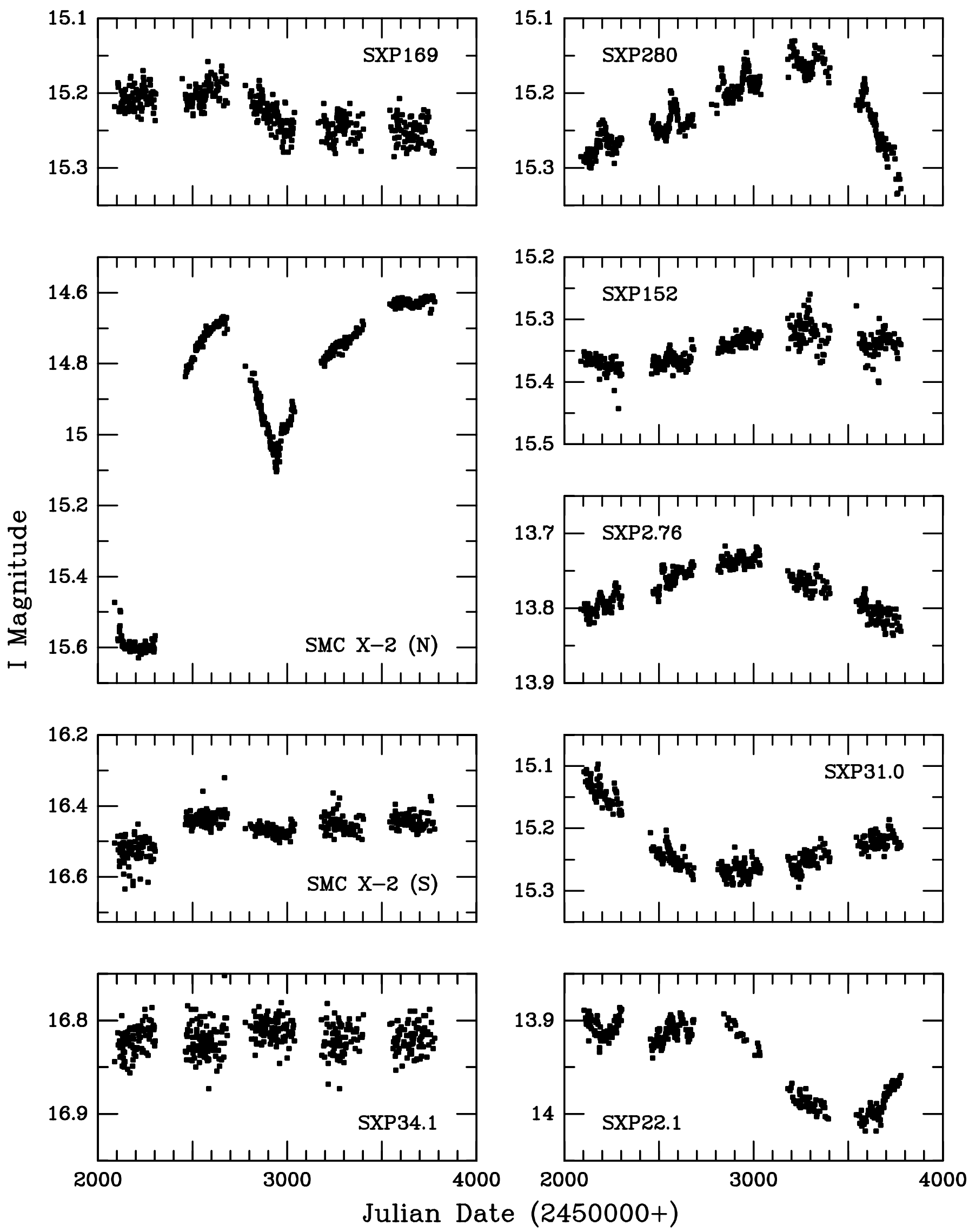

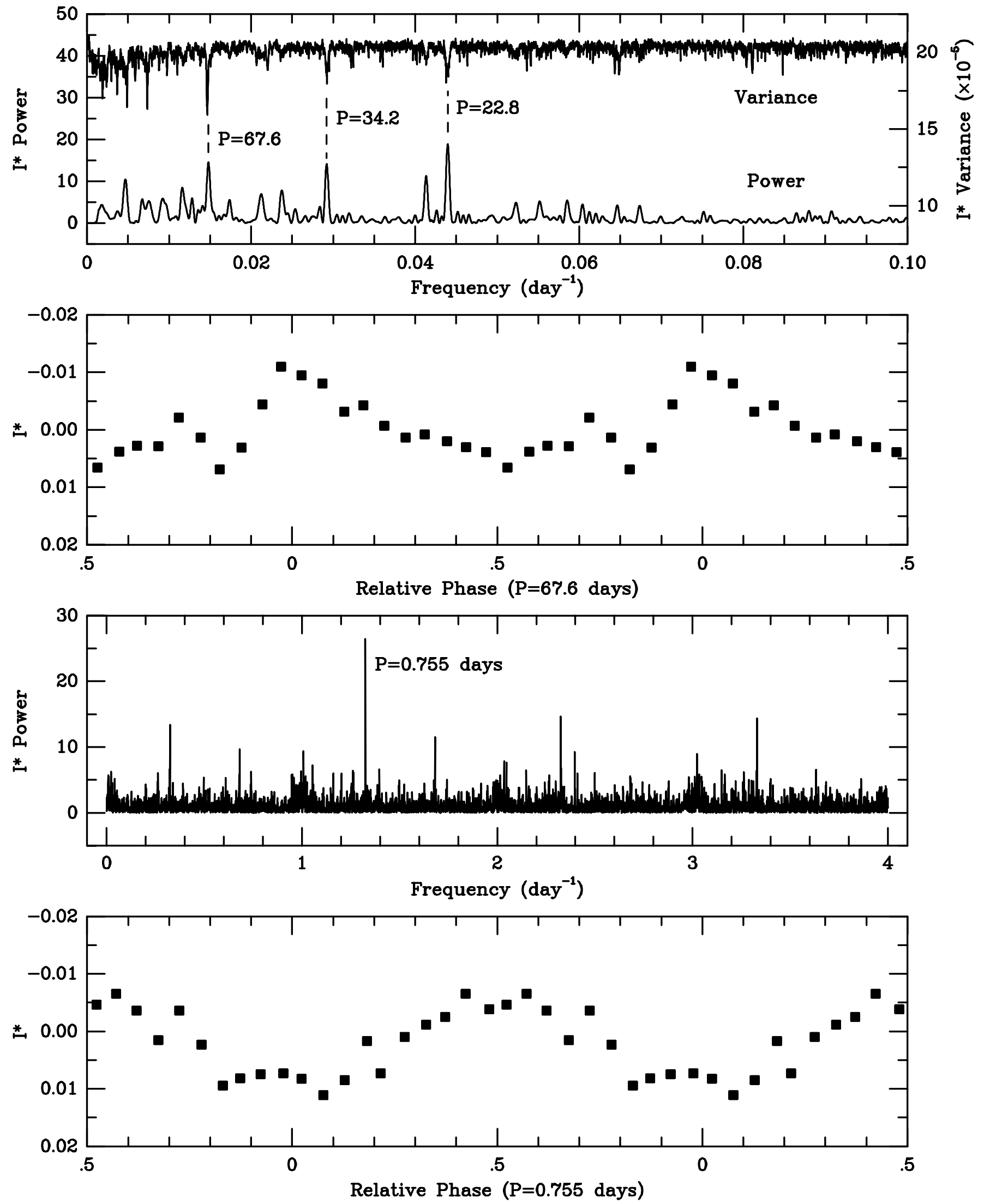


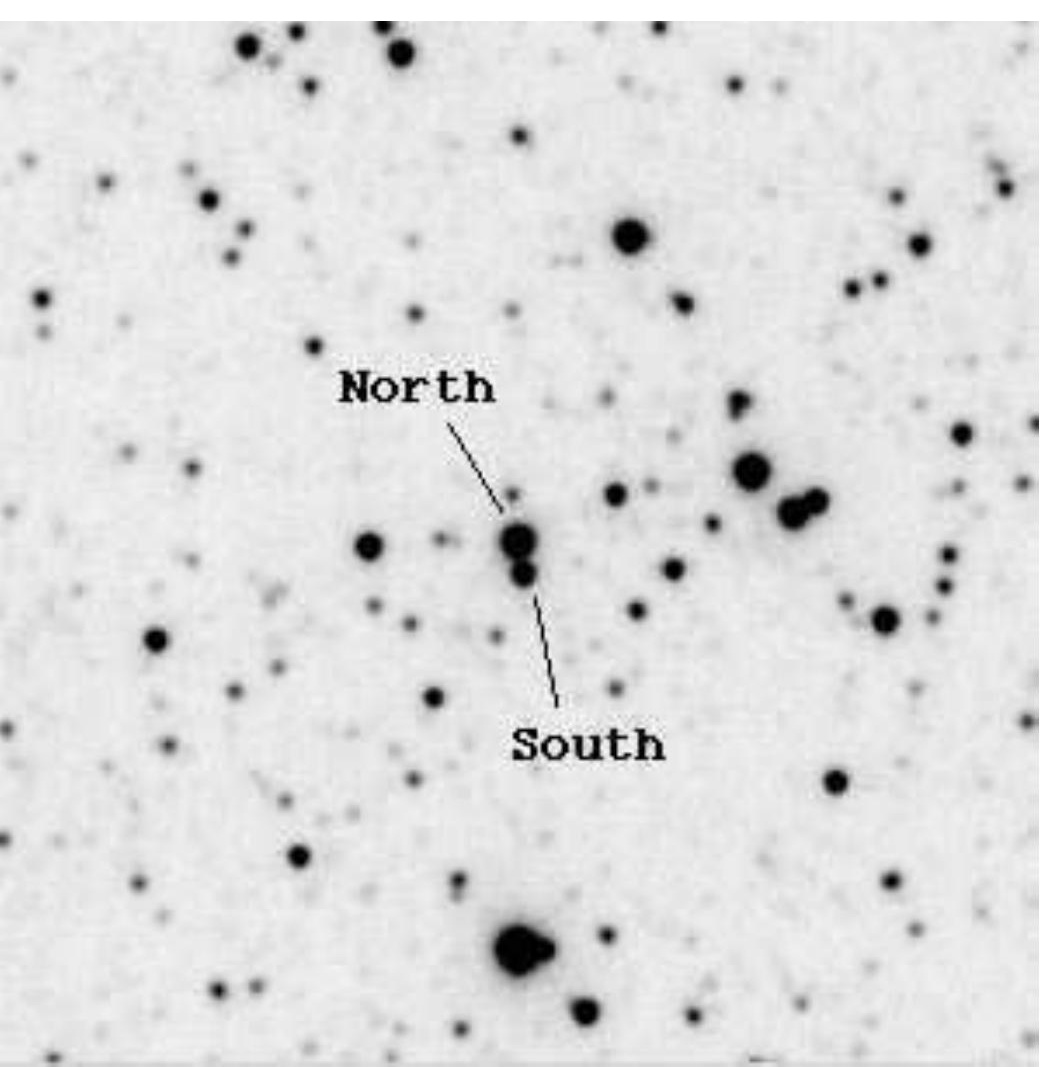



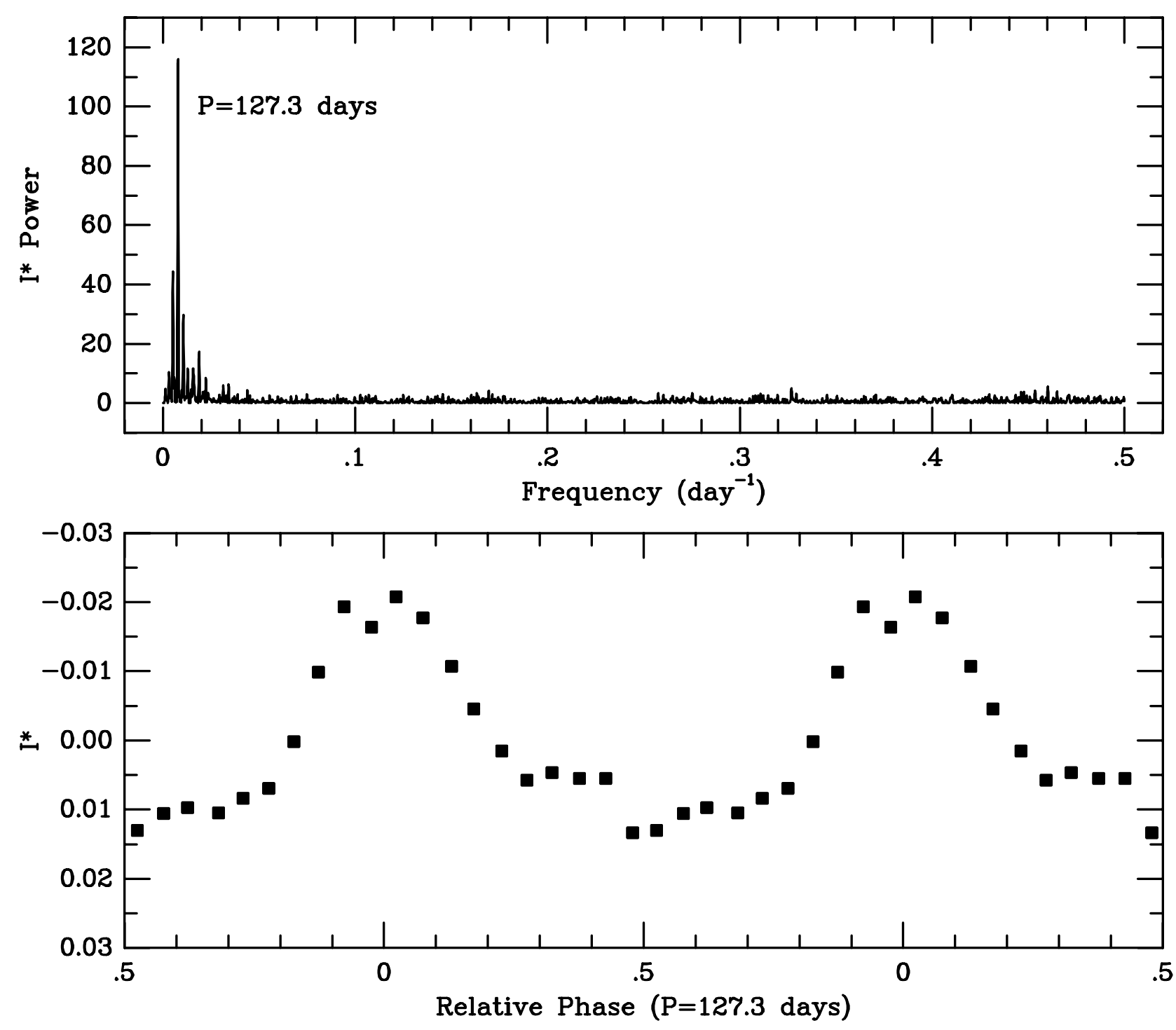

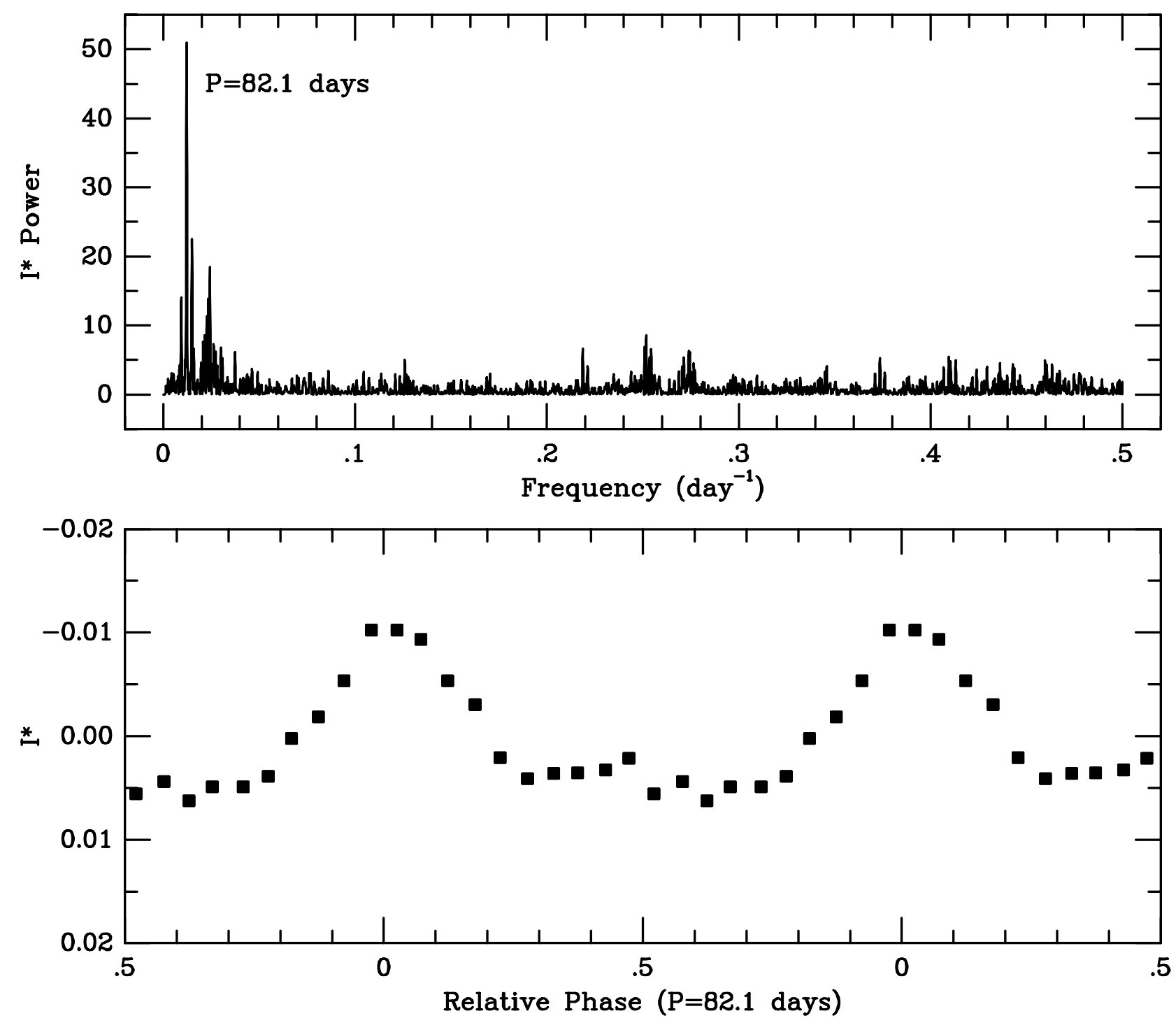

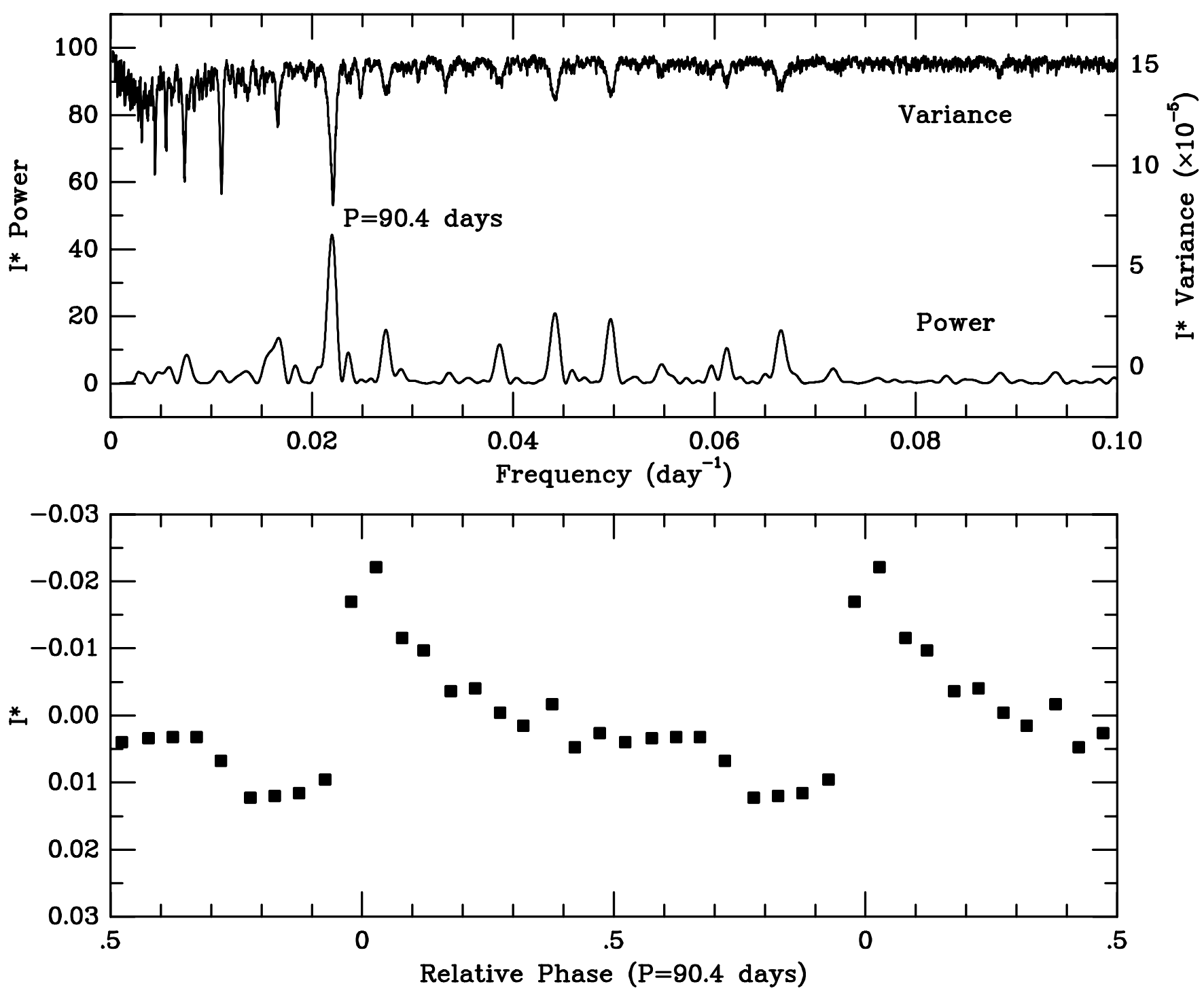